QUARTERLY OF APPLIED MATHEMATICS

VOLUME LXX, NUMBER 2

JUNE 2012, PAGES 199-218

S 0033-569X(2012)01279-8

Article electronically published on February 2, 2012

\title{
NONEXISTENCE OF GLOBAL WEAK SOLUTION WITH ONLY ONE STABLE SUPERSONIC CONIC SHOCK WAVE FOR THE STEADY SUPERSONIC EULER FLOW PAST A PERTURBED CONE
}

BY

XU GANG (Department of Mathematics and IMS, Nanjing University, Nanjing 210093, China)

AND

YIN HUICHENG (Department of Mathematics and IMS, Nanjing University, Nanjing 210093, China)

Abstract. Recently, for the potential equation, a global stable weak solution with only one conic shock wave has been established in some references. However, in contrast to the case of the potential equation, due to the essential influence of the rotations for the Euler flow, in this paper we will show that the global weak solution of the Euler system with one stable supersonic conic shock wave does not exist when a uniform supersonic incoming flow hits an infinitely long and curved sharp conic body.

1. Introduction and main results. In this paper we study the supersonic conic shock wave problem for the 3-D steady full Euler system when a uniform supersonic incoming flow hits an infinitely long and curved sharp conic body. The steady full Euler system is described as

$$
\begin{cases}\sum_{j=1}^{3} \partial_{j}\left(\rho u_{j}\right)=0 & \text { (Conservation of mass) } \\ \sum_{j=1}^{3} \partial_{j}\left(\rho u_{i} u_{j}\right)+\partial_{i} P=0, \quad i=1,2,3, & \text { (Conservation of momentum) } \\ \sum_{j=1}^{3} \partial_{j}\left(\left(\rho e+\frac{1}{2} \rho|u|^{2}+P\right) u_{j}\right)=0 & \text { (Conservation of energy) } \\ P=P(\rho, S), \quad e=e(\rho, S) & \text { (Equations of state) }\end{cases}
$$

Received February 12, 2010.

2010 Mathematics Subject Classification. Primary 35L70, 35L65, 35L67, 76N15.

Key words and phrases. Supersonic flow, conic shock, full Euler system, stream line, nonexistence.

This project is supported by the National Natural Science Foundation of China (Nos.10931007, 10871096, 11025105), the Doctorial Program Foundation of Ministry of Education of China (No.20090091110005) and Natural Science Foundation of Jiangsu province (10KJB110002).

E-mail address: gxu@ujs.edu.cn

E-mail address: huicheng@nju.edu.cn 


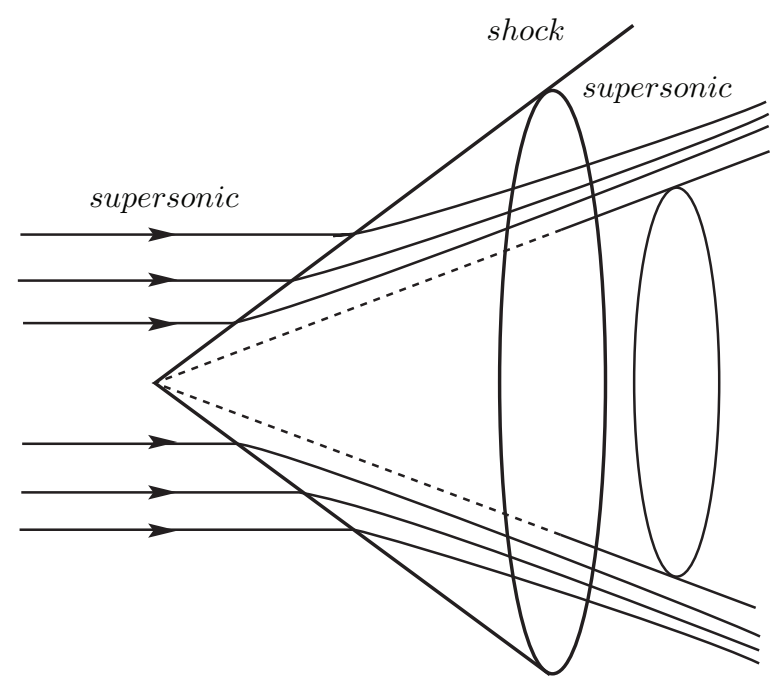

FIG. 1

where $u=\left(u_{1}, u_{2}, u_{3}\right), \rho, P, e$ and $S$ stand for the velocity, density, pressure, internal energy and specific entropy, respectively. Moreover, the pressure function $P=P(\rho, S)$ and the internal energy function $e=e(\rho, S)$ are smooth in their arguments, which satisfy $\partial_{\rho} P(\rho, S)>0$ and $\partial_{S} e(\rho, S)>0$ for $\rho>0$. In addition, $c(\rho, S)=\sqrt{\partial_{\rho} P(\rho, S)}$ stands for the local sound speed.

For the ideal polytropic gases, the equations of state are given by

$$
P=A \rho^{\gamma} e^{\frac{S}{c_{v}}} \quad \text { and } \quad e=\frac{P}{(\gamma-1) \rho} ;
$$

here $A, c_{v}$ and $\gamma$ are positive constants and $1<\gamma<3$.

Given a uniform supersonic incoming flow $\left(0,0, q_{0}\right)$ with constant density $\rho_{0}>0$ and constant pressure $P_{0}$, if this flow hits the circular cone $\sqrt{x_{1}^{2}+x_{2}^{2}}=b_{0} x_{3}$ along the axis in the $x_{3}$ direction (see Figure 1), then as illustrated on the pages 406-408 of book [4], there will appear a global supersonic conic shock $\sqrt{x_{1}^{2}+x_{2}^{2}}=s_{0} x_{3}\left(s_{0}>b_{0}\right)$ attached at the tip of the cone when $b_{0}$ is less than a critical value $b^{*}\left(b^{*}\right.$ is determined by the parameters of the incoming flow). When the surface of the cone is perturbed, we want to know whether the globally stable structure with only one multidimensional supersonic conic shock can be kept or not. The purpose of this paper is to show that such a globally stable structure does not exist.

We note that there have been many studies on the local or global supersonic conic shock problems such as in [2]-6], 8], 13] and the references therein. More concretely, for the 3-D unsteady potential equation, the author in [8] has shown the global existence of a conic shock wave solution under the suitable boundary condition on the conic surface. With respect to the attached supersonic conic shock problem for the 3-D steady potential equation, under the different assumptions on the Mach number of the supersonic incoming flow and the vertex angle of the conic body, the authors in [3], [5] - 6] and [13] have established the global existence and stability, respectively, of a conic shock. For the 3-D 
full Euler system, under some certain restrictions on the perturbed conic bodies, the local supersonic conic shocks have been studied in 2]. However, so far it is unknown whether there exists a globally stable weak solution with only one multidimensional supersonic conic shock for the 3-D full Euler system when the supersonic flow hits a curved sharp cone. Next, our focus is to answer this problem.

Suppose that the supersonic incoming flow with state $\left(\rho_{0}, 0,0, q_{0}, P_{0}\right)$ hits the perturbed conic body along the $x_{3}$ direction, whose surface equation is denoted by $r=b\left(x_{3}\right)$; here $r=\sqrt{x_{1}^{2}+x_{2}^{2}}, b\left(x_{3}\right)=b_{0} x_{3}+\varepsilon \varphi\left(x_{3}\right), \varepsilon>0$ sufficiently small, $\varphi\left(x_{3}\right) \not \equiv 0$ and $\varphi\left(x_{3}\right) \in C_{0}^{\infty}(0, l)$ with some fixed positive number $l>0$. In addition, $b_{0}>0$ is suitably small such that the resulting supersonic shock will be attached at the vertex of the conic body.

By the symmetric property of the perturbed conic surface, it is convenient to introduce the following cylindrical coordinates $\left(x_{3}, r\right)$ to study our problem:

$$
r=\sqrt{x_{1}^{2}+x_{2}^{2}}, \quad x_{3}=x_{3} .
$$

For the polytropic gas and axisymmetric solution of (1.1), i.e., $\left(\rho(x), u_{1}(x), u_{2}(x)\right.$, $\left.u_{3}(x), P(x)\right) \equiv\left(\rho\left(x_{3}, r\right), U\left(x_{3}, r\right) \frac{x_{1}}{r}, U\left(x_{3}, r\right) \frac{x_{2}}{r}, u_{3}\left(x_{3}, r\right), P\left(x_{3}, r\right)\right)$, then (1.1) can be simplified as

$$
\left\{\begin{array}{l}
\partial_{r}(r \rho U)+\partial_{3}\left(r \rho u_{3}\right)=0 \\
\partial_{r}\left(r \rho U^{2}\right)+\partial_{3}\left(r \rho U u_{3}\right)+r \partial_{r} P=0 \\
\partial_{r}\left(r \rho U u_{3}\right)+\partial_{3}\left(r \rho u_{3}^{2}\right)+r \partial_{3} P=0 \\
\partial_{r}\left(r \rho U\left(\frac{1}{2}\left(U^{2}+u_{3}^{2}\right)+\frac{\gamma P}{(\gamma-1) \rho}\right)\right)+\partial_{3}\left(r \rho u_{3}\left(\frac{1}{2}\left(U^{2}+u_{3}^{2}\right)+\frac{\gamma P}{(\gamma-1) \rho}\right)\right)=0 .
\end{array}\right.
$$

Suppose that the $C^{1}$ flow field behind the supersonic shock $r=\chi\left(x_{3}\right)$ with $\chi(0)=0$ is denoted by $\left(\rho^{+}\left(x_{3}, r\right), U^{+}\left(x_{3}, r\right), u_{3}^{+}\left(x_{3}, r\right), P^{+}\left(x_{3}, r\right)\right)$. Then, in the domain $\Omega_{+}=$ $\left\{\left(x_{3}, r\right): x_{3}>0, b\left(x_{3}\right)<r<\chi\left(x_{3}\right)\right\}$, it follows from (1.3) that $\left(\rho^{+}, U^{+}, u_{3}^{+}, P^{+}\right)$satisfies:

$$
\left\{\begin{array}{l}
\partial_{r}\left(r \rho^{+} U^{+}\right)+\partial_{3}\left(r \rho^{+} u_{3}^{+}\right)=0 \\
\partial_{r}\left(r \rho^{+}\left(U^{+}\right)^{2}\right)+\partial_{3}\left(r \rho^{+} U^{+} u_{3}^{+}\right)+r \partial_{r} P^{+}=0 \\
\partial_{r}\left(r \rho^{+} U^{+} u_{3}^{+}\right)+\partial_{3}\left(r \rho^{+}\left(u_{3}^{+}\right)^{2}\right)+r \partial_{3} P^{+}=0
\end{array}\right.
$$

and

$$
\left(U^{+} \partial_{r}+u_{3}^{+} \partial_{3}\right)\left(\frac{1}{2}\left(\left(U^{+}\right)^{2}+\left(u_{3}^{+}\right)^{2}\right)+\frac{\gamma P^{+}}{(\gamma-1) \rho^{+}}\right)=0
$$

with $\left(U^{+}\right)^{2}+\left(u_{3}^{+}\right)^{2}>c^{2}\left(\rho^{+}, S^{+}\right)$.

On the shock $r=\chi\left(x_{3}\right)$, the Rankine-Hugoniot conditions imply

$$
\left\{\begin{array}{l}
{[\rho U]-\chi^{\prime}\left(x_{3}\right)\left[\rho u_{3}\right]=0} \\
{\left[P+\rho U^{2}\right]-\chi^{\prime}\left(x_{3}\right)\left[\rho U u_{3}\right]=0} \\
{\left[\rho U u_{3}\right]-\chi^{\prime}\left(x_{3}\right)\left[P+\rho u_{3}^{2}\right]=0}
\end{array}\right.
$$

and

$$
\left[\rho U\left(\frac{1}{2}\left(U^{2}+u_{3}^{2}\right)+\frac{\gamma P}{(\gamma-1) \rho}\right)\right]-\chi^{\prime}\left(x_{3}\right)\left[\rho u_{3}\left(\frac{1}{2}\left(U^{2}+u_{3}^{2}\right)+\frac{\gamma P}{(\gamma-1) \rho}\right)\right]=0 .
$$


It is noted that combining the first equality in (1.6) with (1.7) and (1.5) yields

$$
\frac{1}{2}\left(\left(U^{+}\right)^{2}+\left(u_{3}^{+}\right)^{2}\right)+\frac{\gamma P^{+}}{(\gamma-1) \rho^{+}}=\frac{1}{2} q_{0}^{2}+\frac{\gamma P_{0}}{(\gamma-1) \rho_{0}} \equiv C_{0} .
$$

In addition, the Lax geometrical entropy condition (see Chapter 17 of [11, and so on) holds true:

$$
\left\{\begin{array}{l}
\lambda_{2}\left(x_{3}, \chi\left(x_{3}\right)\right)<\chi^{\prime}\left(x_{3}\right)<\lambda_{3}\left(x_{3}, \chi\left(x_{3}\right)\right) ; \\
\frac{c\left(\rho_{0}, S_{0}\right)}{\sqrt{q_{0}^{2}-c^{2}\left(\rho_{0}, S_{0}\right)}}<\chi^{\prime}\left(x_{3}\right),
\end{array}\right.
$$

where $\lambda_{i}\left(x_{3}, \chi\left(x_{3}\right)\right)=\left.\lambda_{i}\left(\rho^{+}\left(x_{3}, r\right), U^{+}\left(x_{3}, r\right), u_{3}^{+}\left(x_{3}, r\right), P^{+}\left(x_{3}, r\right)\right)\right|_{r=\chi\left(x_{3}\right)}(1 \leq i \leq 3)$; more concretely,

$$
\lambda_{1,3}\left(\rho, U, u_{3}, P\right)=\frac{U u_{3} \mp c(\rho, S) \sqrt{U^{2}+u_{3}^{2}-c^{2}(\rho, S)}}{u_{3}^{2}-c^{2}(\rho, S)}
$$

and $\lambda_{2}\left(\rho, U, u_{3}, S\right)=\frac{U}{u_{3}}$, which are three eigenvalues of the characteristic matrix

$$
\lambda\left(\begin{array}{ccc}
u_{3} & 0 & \rho \\
0 & u_{3} & 0 \\
\frac{c^{2}}{\rho} & 0 & u_{3}
\end{array}\right)-\left(\begin{array}{ccc}
U & \rho & 0 \\
\frac{c^{2}}{\rho} & U & 0 \\
0 & 0 & U
\end{array}\right)
$$

of (1.4). Here it is noted that (1.4) is strictly hyperbolic with respect to the $x_{3}$ direction in our paper.

Due to the fixed wall condition, we have on the conic surface $r=b\left(x_{3}\right)$,

$$
U^{+}=b^{\prime}\left(x_{3}\right) u_{3}^{+} \text {. }
$$

The main result in our paper is:

Theorem 1.1 (Nonexistence of one globally stable supersonic conic shock). Under the assumptions above, for suitably small $b_{0}$, there exists an $\varepsilon_{0}>0$ such that for $0<\varepsilon<$ $\varepsilon_{0}$, the problem (1.4) with (1.6) and (1.8)-(1.10) has no global solution $\left(\rho^{+}(x), U^{+}(x)\right.$, $\left.u_{3}^{+}(x), P^{+}(x) ; \chi\left(x_{3}\right)\right)$ in $\Omega_{+}$which admits the following properties:

(i). $\chi\left(x_{3}\right) \in C^{2}[0, \infty)$, and

$$
\left\|\chi^{\prime}\left(x_{3}\right)-s_{0}\right\|_{L^{\infty}[0, \infty)} \leq \delta,
$$

where here and below $\delta>0$ is a suitably small quantity as $\varepsilon<\varepsilon_{0}$.

(ii). $\left(\rho^{+}, U^{+}, u_{3}^{+}, P^{+}\right) \in C^{1}\left(\bar{\Omega}_{+} \backslash(0,0)\right) \cap L^{\infty}\left(\Omega_{+}\right)$, and

$$
\left\|\left(\rho^{+}, U^{+}, u_{3}^{+}, P^{+}\right)\left(x_{3}, r\right)-\left(\hat{\rho}, \hat{U}, \hat{u}_{3}, \hat{P}\right)\left(\frac{r}{x_{3}}\right)\right\|_{C^{1}\left(\bar{\Omega}_{+}\right)} \leq \delta
$$

here $\left\|g\left(x_{3}, r\right)\right\|_{C^{1}\left(\bar{\Omega}_{+}\right)} \equiv\|g\|_{L^{\infty}\left(\bar{\Omega}_{+}\right)}+\left\|\nabla_{x_{3}, r} g\right\|_{L^{\infty}\left(\bar{\Omega}_{+}\right)},\left(\hat{\rho}\left(\frac{r}{x_{3}}\right), \hat{U}\left(\frac{r}{x_{3}}\right), \hat{u}_{3}\left(\frac{r}{x_{3}}\right), \hat{P}\left(\frac{r}{x_{3}}\right)\right)$ stands for the extension of the self-similar downstream supersonic state $\left(\tilde{\rho}\left(\frac{r}{x_{3}}\right), \tilde{U}\left(\frac{r}{x_{3}}\right), \tilde{u}_{3}\left(\frac{r}{x_{3}}\right), \tilde{P}\left(\frac{r}{x_{3}}\right)\right)$ behind the shock $r=s_{0} x_{3}$, which is formed for the supersonic incoming flow $\left(\rho_{0}, 0,0, q_{0}, P_{0}\right)$ past the cone $\left\{x: \sqrt{x_{1}^{2}+x_{2}^{2}} \leq b_{0} x_{3}\right\}$. For more detailed information on $\left(\tilde{\rho}\left(\frac{r}{x_{3}}\right), \tilde{U}\left(\frac{r}{x_{3}}\right), \tilde{u}_{3}\left(\frac{r}{x_{3}}\right), \tilde{P}\left(\frac{r}{x_{3}}\right)\right)$ and $\left(\hat{\rho}\left(\frac{r}{x_{3}}\right), \hat{U}\left(\frac{r}{x_{3}}\right), \hat{u}_{3}\left(\frac{r}{x_{3}}\right), \hat{P}\left(\frac{r}{x_{3}}\right)\right)$, one can see Lemma 2.1-Lemma 2.2 and Remark 2.1 in $\S 2$ below. 
REMARK 1.1. Due to $b\left(x_{3}\right)-b_{0} x_{3}=\varepsilon \varphi\left(x_{3}\right) \in C_{0}^{\infty}(0, l)$, there exists a small neighborhood $B\left(0, \delta_{0}\right)$ of the origin $O=(0,0,0)$ such that the system (1.4)-(1.5) can be reduced to an ordinary differential system in the variable $s=\frac{r}{x_{3}}$ (see (2.1) in $\S 2$ below) and further reduces $\left(\rho^{+}, U^{+}, u_{3}^{+}, P^{+} ; \chi\left(x_{3}\right)\right) \equiv\left(\tilde{\rho}^{+}, \tilde{U}^{+}, \tilde{u}_{3}^{+}, \tilde{P}^{+} ; s_{0} x_{3}\right)$ in the region $\Omega_{+} \cap B\left(0, \delta_{0}\right)$. In addition, the system (1.4) is symmetrizable hyperbolic with respect to the $x_{3}$ direction. These, together with the local existence and stability of a multidimensional shock wave in [12] or the Appendix of [8, we know that (i) and (ii) of Theorem 1.1 are reasonable in the bounded subdomain of $\Omega_{+}$. In particular, in the potential equation case, (i) and (ii) of Theorem 1.1 have been shown and $\delta \sim \varepsilon$ is also obtained in [3], [5]-6] and [13].

REMARK 1.2. Our result illustrates the essential differences between the 3-D full Euler system and the 3 -D potential equation when one studies the global supersonic conic shock problem, which is induced by the rotation $\operatorname{curl} u \equiv\left(\partial_{2} u_{3}-\partial_{3} u_{2}, \partial_{3} u_{1}-\partial_{1} u_{3}, \partial_{1} u_{2}-\partial_{2} u_{1}\right)$. In the case of the potential equation, due to curl $u \equiv 0$, then based on this, the full Euler system can be reduced into a second-order nonlinear wave equation, and the stability of a global supersonic conic shock can be established as in [3] and [5]-6].

Although there are many well-known blow-up results of smooth solutions to the multidimensional compressible Euler system when the suitable Cauchy initial values are posed (see the references [1, [7, [9]-[10], [15], and so on), due to the influences of the fixed conic boundary and the shock surface in our paper, it seems that some methods and techniques used in those references cannot be available for us. In order to prove Theorem 1.1, our key observation is that the supersonic shock curve must be straight under the assumptions of Theorem 1.1. Subsequently, we can derive that the downstream supersonic solution must be the background solution $\left(\tilde{\rho}\left(\frac{r}{x_{3}}\right), \tilde{U}\left(\frac{r}{x_{3}}\right), \tilde{u}_{3}\left(\frac{r}{x_{3}}\right), \tilde{P}\left(\frac{r}{x_{3}}\right)\right)$ by use of the uniqueness result of solutions to hyperbolic equations, and further derive that the perturbed conic surface is just only $r=b_{0} x_{3}$. However, this is obviously a contradiction with the property of $b\left(x_{3}\right) \not \equiv b_{0} x_{3}$. From this, we complete the proof of Theorem 1.1.

Our paper is organized as follows. In $\S 2$, for the full Euler system, we will show that there exists an attached supersonic conic shock $r=s_{0} x_{3}$ when a uniform supersonic incoming flow $\left(\rho_{0}, 0,0, q_{0}, P_{0}\right)$ hits the sharp cone $r=b_{0} x_{3}$. In $\S 3$, under the assumptions of Theorem 1.1, it is shown that a globally stable supersonic shock curve must be straight. In $\S 4$, by use of the standard theory on the second-order quasilinear hyperbolic equations, we prove that the downstream supersonic solution is the same as the background solution and further derive that the perturbed conic surface $r=b\left(x_{3}\right)$ is $r=b_{0} x_{3}$. By this contradiction, Theorem 1.1 can be shown.

2. Self-similar supersonic background shock solution. Suppose that there is a uniform supersonic flow $\left(\rho_{0}, 0,0, q_{0}, P_{0}\right)$ and this flow hits the circular cone $r=b_{0} x_{3}$ along the $x_{3}$ direction. Then as described in [4, there exists a critical value $b^{*}$ such that there will appear an attached supersonic conic shock $r=s_{0} x_{3}\left(s_{0}>b_{0}\right)$ for $b_{0}<b^{*}$. Moreover the solution of (1.4)-(1.5) has such a form: $\rho^{+}(x)=\tilde{\rho}(s), u_{1}^{+}(x)=\tilde{U}(s) \frac{x_{1}}{r}, u_{2}^{+}(x)=$ $\tilde{U}(s) \frac{x_{2}}{r}, u_{3}^{+}(x)=\tilde{u}_{3}(s)$ and $P^{+}(x)=\tilde{P}(s)$ with $s=\frac{r}{x_{3}}$. With respect to the existence of $\left(\tilde{\rho}(s), \tilde{U}(s), \tilde{u}_{3}(s), \tilde{P}(s) ; s_{0}\right)$, Sect.154-Sect.156 of [4 have given the outline of a proof procedure. However, for the reader's convenience and the use later on, we will give a 
detailed proof and further establish some precise properties on the background supersonic solution $\left(\tilde{\rho}(s), \tilde{U}(s), \tilde{u}_{3}(s), \tilde{P}(s)\right)$.

By (1.3) and a direct computation, we obtain that $\left(\tilde{\rho}(s), \tilde{U}(s), \tilde{u}_{3}(s), \tilde{P}(s)\right)$ satisfies

$$
\left\{\begin{array}{l}
\tilde{\rho}^{\prime}(s)=-\frac{\tilde{\rho} \tilde{U}\left(s \tilde{u}_{3}-\tilde{U}\right)}{s\left(\left(1+s^{2}\right) c^{2}(s)-\left(s \tilde{u}_{3}-\tilde{U}\right)^{2}\right)}, \\
\tilde{U}^{\prime}(s)=-\frac{c^{2}(s) \tilde{U}}{s\left(\left(1+s^{2}\right) c^{2}(s)-\left(s \tilde{u}_{3}-\tilde{U}\right)^{2}\right)}, \\
\tilde{u}_{3}^{\prime}(s)=\frac{c^{2}(s) \tilde{U}}{\left(1+s^{2}\right) c^{2}(s)-\left(s \tilde{u}_{3}-\tilde{U}\right)^{2}}, \\
\tilde{P}^{\prime}(s)=-\frac{\tilde{\rho} c^{2}(s) \tilde{U}\left(s \tilde{u}_{3}-\tilde{U}\right)}{s\left(\left(1+s^{2}\right) c^{2}(s)-\left(s \tilde{u}_{3}-\tilde{U}\right)^{2}\right)},
\end{array}\right.
$$

here $\tilde{U}^{2}(s)+\tilde{u}_{3}^{2}(s)>c^{2}(s) \equiv \frac{\gamma \tilde{P}(s)}{\tilde{\rho}(s)}$. Moreover, Bernoulli's law holds true:

$$
\frac{1}{2}\left(\tilde{U}^{2}(s)+\tilde{u}_{3}^{2}(s)\right)+\frac{c^{2}(s)}{\gamma-1} \equiv C_{0} .
$$

For notational convenience, below we will drop " " in the solution $\left(\tilde{\rho}(s), \tilde{U}(s), \tilde{u}_{3}(s)\right.$, $\tilde{P}(s))$.

According to Lemma 2.2 below, we know the denominator $\left(1+s^{2}\right) c^{2}(s)-\left(s u_{3}-U\right)^{2}>0$ in (2.1) for $b_{0} \leq s \leq s_{0}$. This means that the ODE system (2.1) is meaningful.

On the shock surface $r=s_{0} x_{3}$, due to the Rankine-Hugoniot conditions and Lax's geometrical entropy condition, one has

$$
\left\{\begin{array}{l}
{[\rho U]-s_{0}\left[\rho u_{3}\right]=0} \\
{\left[P+\rho U^{2}\right]-s_{0}\left[\rho U u_{3}\right]=0} \\
{\left[\rho U u_{3}\right]-s_{0}\left[P+\rho u_{3}^{2}\right]=0}
\end{array}\right.
$$

and

$$
\left\{\begin{array}{l}
\lambda_{2}\left(\rho\left(s_{0}\right), U\left(s_{0}\right), u_{3}\left(s_{0}\right), P\left(s_{0}\right)\right)<s_{0}<\lambda_{3}\left(\rho\left(s_{0}\right), U\left(s_{0}\right), u_{3}\left(s_{0}\right), P\left(s_{0}\right)\right) \\
\frac{c\left(\rho_{0}, S_{0}\right)}{\sqrt{q_{0}^{2}-c^{2}\left(\rho_{0}, S_{0}\right)}}<s_{0} .
\end{array}\right.
$$

Additionally,

$$
U\left(b_{0}\right)=b_{0} u_{3}\left(b_{0}\right)
$$

With respect to the nonlinear system (2.1)-(2.2) with free boundary value conditions (2.3)-(2.4) and fixed boundary value condition (2.5), we have

Lemma 2.1. For suitably small $b_{0}>0$, we can conclude that (2.1)-(2.5) has a smooth solution $\left(\rho(s), U(s), u_{3}(s), P(s)\right)$ and admits a determined shock position $s=s_{0}$ such that $u_{3}(s)>c(s)$ holds true for $b_{0} \leq s \leq s_{0}$. 
Proof. Set $\rho_{+}=\lim _{s \rightarrow s_{0}-0} \rho(s), U_{+}=\lim _{s \rightarrow s_{0}-0} U(s), u_{3+}=\lim _{s \rightarrow s_{0}-0} u_{3}(s)$ and $P_{+}=$ $\lim _{s \rightarrow s_{0}-0} P(s)$. For convenience, let $\sigma=\frac{1}{s}$. Then (2.1) can be rewritten as

$$
\left\{\begin{aligned}
\rho^{\prime}(\sigma) & =\frac{\rho U\left(u_{3}-\sigma U\right)}{\left(1+\sigma^{2}\right) c^{2}(\sigma)-\left(u_{3}-\sigma U\right)^{2}}, \\
U^{\prime}(\sigma) & =\frac{\sigma c^{2}(\sigma) U}{\left(1+\sigma^{2}\right) c^{2}(\sigma)-\left(u_{3}-\sigma U\right)^{2}}, \\
u_{3}^{\prime}(\sigma) & =-\frac{c^{2}(\sigma) U}{\left(1+\sigma^{2}\right) c^{2}(\sigma)-\left(u_{3}-\sigma U\right)^{2}}, \\
P^{\prime}(\sigma) & =\frac{\rho c^{2}(\sigma) U\left(u_{3}-\sigma U\right)}{\left(1+\sigma^{2}\right) c^{2}(\sigma)-\left(u_{3}-\sigma U\right)^{2}},
\end{aligned}\right.
$$

here $\sigma_{0}=\frac{1}{s_{0}}$.

It follows from a direct computation that

$$
\left(\frac{P}{\rho^{\gamma}}\right)^{\prime}(\sigma)=0
$$

This means that

$$
P(\sigma)=A_{+} \rho^{\gamma}(\sigma)
$$

here $A_{+}=\frac{P_{+}}{\rho_{+}^{\gamma}}$.

By (1.8) and (2.7), we know that $\rho(\sigma), P(\sigma)$ and the sound speed $c(\sigma)$ can be expressed as the smooth functions of $\left(U(\sigma), u_{3}(\sigma)\right)$, respectively. Namely,

$$
\rho(\sigma)=\rho\left(U(\sigma), u_{3}(\sigma)\right), P(\sigma)=P\left(U(\sigma), u_{3}(\sigma)\right) \text { and } c(\sigma)=c\left(U(\sigma), u_{3}(\sigma)\right) .
$$

In addition, by the second and third equation in (2.6), we find that

$$
\frac{U^{\prime}(\sigma)}{u_{3}^{\prime}(\sigma)}=-\sigma
$$

As indicated in Sect.155 of [4], it is particularly amenable to treat $U$ as a function of $u_{3}$. In this case, one has

$$
U^{\prime}\left(u_{3}\right)=-\sigma
$$

and

$$
u_{3}^{\prime}(\sigma)=-\frac{1}{U^{\prime \prime}\left(u_{3}\right)}, \quad U^{\prime}(\sigma)=-\frac{U^{\prime}\left(u_{3}\right)}{U^{\prime \prime}\left(u_{3}\right)} .
$$

Substituting (2.10) into the second equation of (2.6) and using (2.7)-(2.8) yield

$$
U U^{\prime \prime}\left(u_{3}\right)=1+\left(U^{\prime}\left(u_{3}\right)\right)^{2}-\frac{\left(u_{3}+U U^{\prime}\left(u_{3}\right)\right)^{2}}{c^{2}} .
$$

Assume that the parameter equation of the shock polar is:

$$
u_{3}=u_{3}(t), U=U(t)
$$

whose equation describes the relation of $u_{3}$ and $U$ on the $\left(u_{3}, U\right)$-plane in terms of the R$\mathrm{H}$ conditions (2.3). Since the algebraic relations (2.3) together with (2.2) are completely analogous to those for the 2-D supersonic uniform flow past a sharp wedge, then its picture can be drawn as the shock polar in Figure 2 (one can also see Fig. 43 of page 313 in [4). 


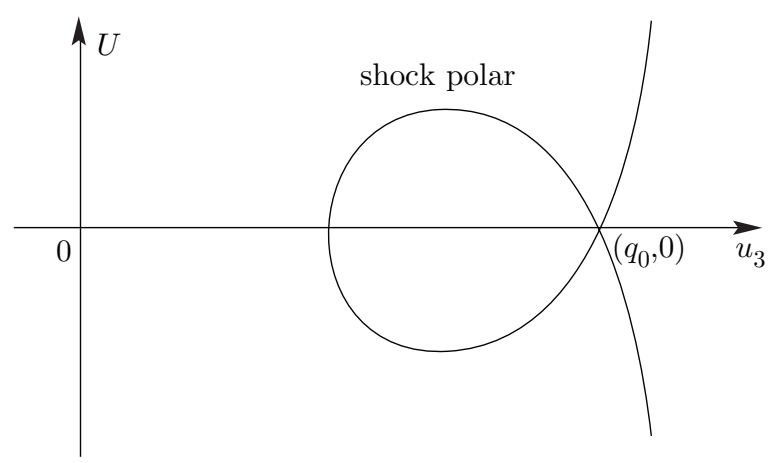

FIG. 2

Next, we discuss the existence of a solution to equation (2.11), which starts from any point $M\left(u_{3}\left(t_{0}\right), U\left(t_{0}\right)\right)$ of the shock polar in the supersonic part. In this case, $U>0, u_{3}>0$ and $\sigma>0$ are only considered.

In light of (2.3), we can look for the related $\sigma_{0}$, which is denoted by $\sigma_{0}\left(t_{0}\right)=$ $\frac{U\left(t_{0}\right)}{q_{0}-u_{3}\left(t_{0}\right)}$ (this equality is derived by the $\mathrm{R}-\mathrm{H}$ conditions (2.3) together with (2.2); the reader is referred to (121.02) on page 306 in [4]). From $(2.9)$, we have $U_{u_{3}}^{\prime}\left(u_{3}\left(t_{0}\right)\right)=$ $-\sigma_{0}\left(t_{0}\right)$.

Now we study the following initial value problem:

$$
\left\{\begin{array}{l}
c^{2} U U^{\prime \prime}\left(u_{3}\right)=\left(c^{2}-U^{2}\right)\left(U^{\prime}\right)^{2}\left(u_{3}\right)-2 u_{3} U U^{\prime}\left(u_{3}\right)+\left(c^{2}-u_{3}^{2}\right), \\
U\left(u_{3}^{0}\right)=U^{0}, U^{\prime}\left(u_{3}^{0}\right)=-\sigma_{0},
\end{array}\right.
$$

where $u_{3}^{0}=u_{3}\left(t_{0}\right), U^{0}=U\left(t_{0}\right)$ and $\sigma_{0}=\sigma_{0}\left(t_{0}\right)$. From the theory of ODE and $U^{0}>0$, (2.12) is locally solvable.

Next, we assert that (2.12) is actually solved in the first quadrant when $\sigma_{0} \leq \sigma \leq \frac{1}{b_{0}}$.

Indeed, in the downstream supersonic domain, the right-hand side of (2.12) is

$$
\left(c^{2}-U^{2}\right)\left(U^{\prime}\right)^{2}\left(u_{3}\right)-2 u_{3} U U^{\prime}\left(u_{3}\right)+\left(c^{2}-u_{3}^{2}\right)=\sigma^{2}\left(\left(1+s^{2}\right) c^{2}-\left(s u_{3}-U\right)^{2}\right)>0 .
$$

From this we derive that $U^{\prime \prime}\left(u_{3}\right)>0$ holds true. By $(2.10)$, one has $u_{3}^{\prime}(\sigma)<0$ and $U^{\prime}(\sigma)>0$; this means that the solution curve of (2.12) extends from southeast to northwest and $U^{\prime}\left(u_{3}\right)$ decreases along the solution curve (but $\left|U^{\prime}\left(u_{3}\right)\right|$ increases) (one can look at Figure 3 for convenience, where $c_{*}=\sqrt{\frac{\gamma-1}{\gamma+1} q_{0}^{2}+\frac{2}{\gamma+1} c^{2}\left(\rho_{0}, S_{0}\right)}$ represents the critical speed). Since $\sigma \in\left[\sigma_{0}, \frac{1}{b_{0}}\right]$, then one can derive that

$$
U-U^{0}=\int_{u_{3}^{0}}^{u_{3}} U_{u_{3}}^{\prime}(z) d z=\int_{u_{3}}^{u_{3}^{0}} \sigma d z \leq \int_{0}^{u_{3}^{0}} \frac{1}{b_{0}} d z \leq \frac{u_{3}^{0}}{b_{0}} .
$$

Thus, $U^{0} \leq U \leq \frac{u_{3}^{0}}{b_{0}}+U^{0}$ holds true. Together with $\left|U_{u_{3}}^{\prime}\right| \leq \frac{1}{b_{0}}$, it follows from the extension theorem of the solution to the ODE that (2.12) is always solvable in the supersonic domain of the first quadrant. On the other hand, for suitably small $b_{0}, u_{3}>c$ 


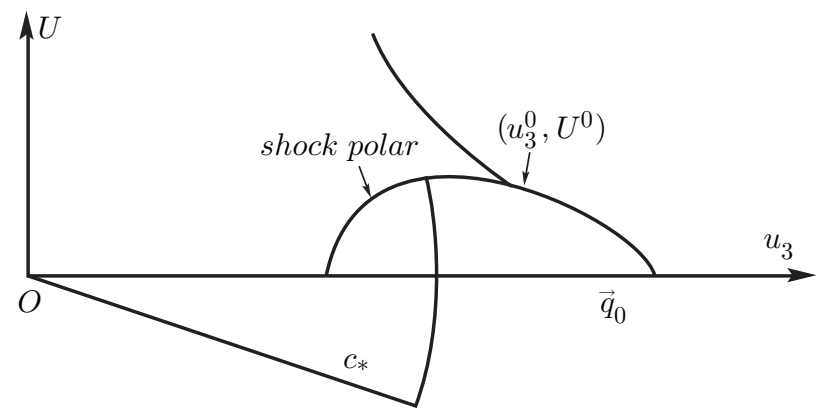

FIG. 3

always holds true (see Lemma 2.2(ii) below). Thus, (2.12) is solved for $\sigma \in\left[\sigma_{0}, \frac{1}{b_{0}}\right]$ and the flow field remains supersonic in the $x_{3}$ direction.

Next, for fixed $t_{0}$, we associate the equation (2.12) with the shock boundary conditions and fixed wall boundary condition in (2.3) and (2.5), respectively. For the requirement of (2.5), we need to look for a point $N$ in the $\left(u_{3}, U\right)$-plane such that the solution curve of (2.12) ends at $N$ and satisfies

$$
\left.\left(u_{3}, U\right)\left(1, U_{u_{3}}^{\prime}\right)\right|_{N}=0
$$

this is equivalent to

$$
\arctan \frac{U}{u_{3}}-\arctan U^{\prime}\left(u_{3}\right)=\frac{\pi}{2} \quad \text { at } \quad N .
$$

Now we show that there exists a predetermined point $N$ such that (2.13) holds.

When point $N$ moves along the solution curve of (2.13)(correspondingly, $\sigma$ increases), then $\arctan \frac{U}{u_{3}}$ increases and $\arctan U^{\prime}\left(u_{3}\right)$ decreases. At $\sigma=\sigma_{0}$, from the R-H condition (2.3), we know that $\frac{U^{0}}{u_{3}^{0}}<k_{\text {shock }}=\frac{1}{\sigma_{0}}=\frac{q_{0}-u_{3}^{0}}{U^{0}}$ (here $k_{\text {shock }}$ stands for the slope of the shock curve in the $\left(x_{3}, r\right)$-plane). This yields

$$
\arctan \frac{U^{0}}{u_{3}^{0}}-\arctan U^{\prime}\left(u_{3}^{0}\right)=\arctan \frac{U^{0}}{u_{3}^{0}}+\arctan \frac{U^{0}}{q_{0}-u_{3}^{0}} \leq \frac{\pi}{2},
$$

where the equality holds if and only if $\left(u_{3}^{0}, U^{0}\right)=\left(q_{0}, 0\right)$. Noting that $\frac{U}{u_{3}} \geq \frac{U^{0}}{u_{3}} \rightarrow+\infty$ and $\arctan \frac{U}{u_{3}} \rightarrow \frac{\pi}{2}$ as $u_{3} \rightarrow 0$, moreover, $-\arctan U_{u_{3}}^{\prime} \geq \arctan \sigma_{0}$ holds. This yields $\arctan \frac{U}{u_{3}}-\arctan U^{\prime}\left(u_{3}\right)>\frac{\pi}{2}$ as $u_{3} \rightarrow 0$. Thus, by the continuity and monotonicity of $\arctan \frac{U}{u_{3}}-\arctan U^{\prime}\left(u_{3}\right)$ with respect to $u_{3}$, there must exist a unique $N$ such that the $\operatorname{arc} M N$ corresponds to the solution of (2.11) together with two boundary values $U\left(u_{3}^{0}\right)=U^{0}$ and $\left.\left(u_{3}, U\right)\left(1, U_{u_{3}}^{\prime}\right)\right|_{N}=0$.

Next, we show that for any sharp body, there exists a unique supersonic shock such that the boundary value problem (2.12) with (2.3) and (2.5) is always solvable.

Indeed, when $M\left(u_{3}\left(t_{0}\right), U\left(t_{0}\right)\right)$ moves at the shock polar in the supersonic domain, it follows from $\left(u_{3}\left(t_{0}\right), U\left(t_{0}\right)\right) \in C^{k}$ and the continuous dependence of the solution on the initial values that $\left.\left(u_{3}, U\right)\right|_{N}$ is $C^{k}$ with respect to the variable $t_{0}$. This continuous curve 


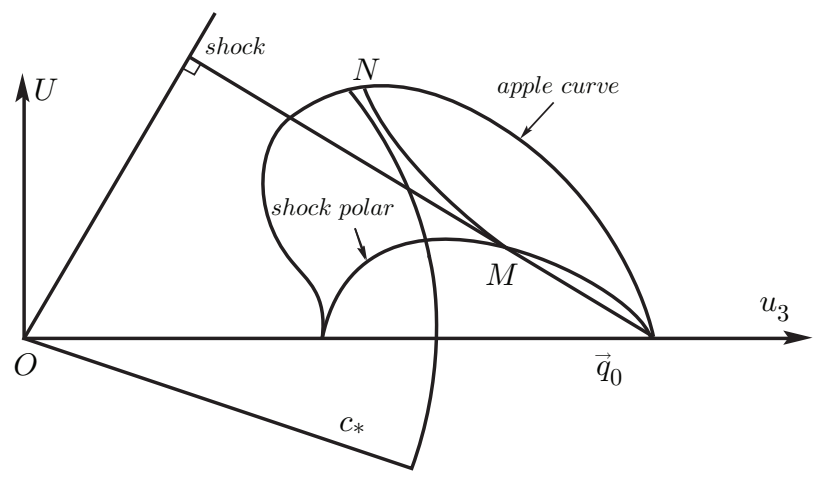

FIG. 4

(together with the transonic shock part), which is formed by point $N$, is called the apple curve in Figure 4 which lies above the shock polar (one can also see the picture in Fig. 8 on page 414 in [4).

If $b_{0} \rightarrow 0$, by use of $\left.U_{u_{3}}^{\prime}\right|_{N}=-\frac{1}{b_{0}}$, then we have $\left.U_{u_{3}}^{\prime}\right|_{N} \rightarrow-\infty$ and $\left.\arctan U_{u_{3}}^{\prime}\right|_{N} \rightarrow$ $-\frac{\pi}{2}$. Hence, it follows from (2.13) and $0<u_{3} \leq u_{3}^{0}<\infty$ that $\left.\arctan \frac{U}{u_{3}}\right|_{N} \rightarrow 0$ and $\left.U\right|_{N} \rightarrow 0$. This implies, when $b_{0}$ is suitably small, that the radial line $U=b_{0} u_{3}$ will intersect with the apple curve in the supersonic part and $u_{3}>c$ holds true. Moreover, by use of (2.13) and the uniqueness theorem of the solution to the ODE, we know that the nonlinear mapping from $N$ to $M$, which is determined by (2.12)-(2.13), is one-toone between the apple curve and the shock polar. Therefore, the supersonic solution of (2.12) exists uniquely when $b_{0}$ is suitably small. This, together with (2.8), yields Lemma 2.1 .

Next, we establish some basic properties on $\left(\rho(s), U(s), u_{3}(s), P(s)\right)$ as in [3], which can be used to illustrate that the system (2.1) makes sense, and treat our nonlinear problem (1.4) with (1.6) and (1.8)-(1.10).

Lemma 2.2. Set $\lambda_{k}(s)=\lambda_{k}\left(\rho(s), U(s), u_{3}(s), P(s)\right), k=1,3$. If $b_{0}$ is suitably small, then for $b_{0} \leq s \leq s_{0}$, the solution $\left(\rho(s), U(s), u_{3}(s), P(s)\right)$ of $(2.1)-(2.5)$ satisfies:

(i). $U^{\prime}(s)<0, u_{3}^{\prime}(s)>0, \rho^{\prime}(s)<0$ and $c^{2}(s)\left(1+s^{2}\right)-\left(s u_{3}(s)-U(s)\right)^{2}>0$.

(ii). $u_{3}(s)>c(s)$.

(iii). $\lambda_{3}(s)>s_{0}$.

Proof. (i). By the R-H conditions (2.3) and Bernoulli's law (2.2), it follows from Sect.121 of [4] that

$$
\left\{\begin{array}{l}
u_{3+}=\left(1-\mu^{2}\right) q_{0} \cos ^{2} \beta+\frac{c_{*}^{2}}{q_{0}} \\
U_{+}=\left(q_{0}-u_{3+}\right) \cot \beta
\end{array}\right.
$$

here $\mu^{2}=\frac{\gamma-1}{\gamma+1}, \beta=\arctan s_{0}$ and $c_{*}^{2}=\mu^{2} q_{0}^{2}+\left(1-\mu^{2}\right) \frac{\gamma P_{0}}{\rho_{0}}$. 
Equivalently, we have

$$
\left\{\begin{array}{l}
u_{3+}=\frac{2 q_{0}}{(\gamma+1)\left(1+s_{0}^{2}\right)}+\frac{(\gamma-1) q_{0}}{\gamma+1}+\frac{2 \gamma P_{0}}{(\gamma+1) \rho_{0} q_{0}} \\
U_{+}=\frac{2 s_{0} q_{0}}{(\gamma+1)\left(1+s_{0}^{2}\right)}-\frac{2 \gamma P_{0}}{(\gamma+1) \rho_{0} q_{0} s_{0}}
\end{array}\right.
$$

Since $U_{+}>0$ holds true, then

$$
0<\frac{\gamma P_{0}}{\rho_{0}}<\frac{s_{0}^{2} q_{0}^{2}}{1+s_{0}^{2}}
$$

By use of the Bernoulli law, we arrive at

$$
\frac{\gamma P_{+}}{\rho_{+}}=\frac{\gamma P_{0}}{\rho_{0}}+\frac{\gamma-1}{2}\left(q_{0}^{2}-u_{3+}^{2}-U_{+}^{2}\right) .
$$

It follows from (2.15)-(2.17) and direct computation that

$$
\begin{aligned}
& \frac{\gamma P_{+}}{\rho_{+}}\left(1+s_{0}^{2}\right)-\left(s_{0} u_{3+}-U_{+}\right)^{2} \\
& \quad=\frac{1}{q_{0}^{2} s_{0}^{2}(1+\gamma)}\left(-2\left(1+s_{0}^{2}\right)^{2}\left(\frac{\gamma P_{0}}{\rho_{0}}\right)^{2}+s_{0}^{2}\left(1+s_{0}^{2}\right) q_{0}^{2}(3-\gamma) \frac{\gamma P_{0}}{\rho_{0}}+q_{0}^{4} s_{0}^{4}(\gamma-1)\right) \\
& \quad=\frac{2\left(1+s_{0}^{2}\right)^{2}}{s_{0}^{2} q_{0}^{2}(1+\gamma)}\left(\frac{s_{0}^{2} q_{0}^{2}}{1+s_{0}^{2}}-\frac{\gamma P_{0}}{\rho_{0}}\right)\left(\frac{\gamma P_{0}}{\rho_{0}}+\frac{(\gamma-1) s_{0}^{2} q_{0}^{2}}{2\left(1+s_{0}^{2}\right)}\right) \\
& \quad>0 .
\end{aligned}
$$

In addition, for the polytropic gas, we have shown that the entropy $S$ behind the shock is a constant $S_{+}$. In this case, the sonic speed can be denoted as $c(s)=c\left(\rho(s), S_{+}\right)$for simplicity.

By the continuity of $\rho(s), U(s)$ and $u_{3}(s)$, (2.18) holds true in $s_{0}-\delta \leq s \leq s_{0}$ with some small $\delta>0$, and then (2.1) makes sense in this interval. Due to (2.1), we know that $\rho^{\prime}(s)<0, U^{\prime}(s)<0, u_{3}^{\prime}(s)>0$ and $P^{\prime}(s)<0$ are valid in $s_{0}-\delta \leq s \leq s_{0}$. On the other hand, we have

$$
\left(c\left(\rho(s), S_{+}\right)-\frac{s u_{3}(s)-U(s)}{\sqrt{1+s^{2}}}\right)^{\prime}=c_{\rho}^{\prime}\left(\rho(s), S_{+}\right) \rho^{\prime}(s)-\frac{s u_{3}^{\prime}(s)-U^{\prime}(s)}{\sqrt{1+s^{2}}}-\frac{u_{3}(s)+s U(s)}{\left(1+s^{2}\right)^{\frac{3}{2}}}<0 .
$$

This means that $c\left(\rho(s), S_{+}\right)-\frac{s u_{3}(s)-U(s)}{\sqrt{1+s^{2}}}$ is a decreasing function of $s$. Thus, we can conclude in $s_{0}-\delta \leq s \leq s_{0}: U(s) \geq U_{+}, \rho(s) \geq \rho_{+}$, and

$$
\begin{aligned}
& \frac{\gamma P(s)}{\rho(s)}\left(1+s^{2}\right)-\left(s u_{3}(s)-U(s)\right)^{2} \\
& =c^{2}\left(\rho(s), S_{+}\right)\left(1+s^{2}\right)-\left(s u_{3}(s)-U(s)\right)^{2} \\
& =\left(1+s^{2}\right)\left(c\left(\rho(s), S_{+}\right)-\frac{s u_{3}(s)-U(s)}{\sqrt{1+s^{2}}}\right)\left(c\left(\rho(s), S_{+}\right)+\frac{s u_{3}(s)-U(s)}{\sqrt{1+s^{2}}}\right) \\
& \geq c\left(\rho_{+}, S_{+}\right)\left(1+b_{0}^{2}\right)\left(c\left(s_{0}\right)-\frac{s_{0} u_{3+}-U_{+}}{\sqrt{1+s_{0}^{2}}}\right)>0 ;
\end{aligned}
$$


here we have used the fact of $s u_{3}(s)-U(s) \geq 0$, which can be easily shown due to $\left(s u_{3}(s)-U(s)\right)^{\prime}=s u_{3}^{\prime}(s)+u_{3}(s)-U^{\prime}(s)>0$ and further $s u_{3}(s)-U(s) \geq b_{0} u_{3}\left(b_{0}\right)-$ $U\left(b_{0}\right)=0$.

From (2.19), one can derive that the denominator in (2.1) is lower bounded away from zero as long as the solution of (2.1) exists. Therefore, (2.19) holds in the whole interval $\left[b_{0}, s_{0}\right]$; meanwhile the solution of (2.1) exists uniquely by the proof procedure of Lemma 2.1 , which satisfies

$$
\rho^{\prime}(s)<0, U^{\prime}(s)<0, u_{3}^{\prime}(s)>0, P^{\prime}(s)<0 .
$$

(ii). First we assert that

$$
\sqrt{\frac{c_{*}^{2}-\mu^{2} q_{0}^{2}}{q_{0}^{2}-c_{*}^{2}}} \leq s_{0} \leq \sqrt{\frac{c_{*}^{2}-\mu^{2} q_{0}^{2}}{q_{0}^{2}-c_{*}^{2}}}+\frac{1}{2}\left(1-\mu^{2}\right) q_{0}^{4} b_{0}+O\left(b_{0}^{2}\right) .
$$

In fact, by (2.14), we have

$$
U_{+}=\left(q_{0}-u_{3+}\right) \sqrt{\frac{q_{0} u_{3+}-c_{*}^{2}}{\left(1-\mu^{2}\right) q_{0}^{2}-q_{0} u_{3+}+c_{*}^{2}}} .
$$

This, together with the R-H conditions (2.3), yields

$$
s_{0}=\frac{q_{0}-u_{3+}}{U_{+}}=\sqrt{\frac{\left(1-\mu^{2}\right) q_{0}^{2}-q_{0} u_{3+}+c_{*}^{2}}{q_{0} u_{3+}-c_{*}^{2}}} .
$$

On the other hand, it follows from a direct computation that

$$
\frac{d s_{0}^{2}}{d u_{3+}}=-\frac{\left(1-\mu^{2}\right) q_{0}^{3}}{\left(q_{0} u_{3+}-c_{*}^{2}\right)^{2}}<0 .
$$

This means that $s_{0}$ is a decreasing function of $u_{3+}$. Moreover, $s_{0}$ achieves its minimum when $u_{3+}=q_{0}$, which is denoted by $s_{0}^{\min }$ and

$$
s_{0}^{\min }=\sqrt{\frac{c_{*}^{2}-\mu^{2} q_{0}^{2}}{q_{0}^{2}-c_{*}^{2}}} .
$$

Due to Lemma 2.2(i) and the fixed wall condition, one has

$$
U_{+} \leq U\left(b_{0}\right)=b_{0} u_{3}\left(b_{0}\right) \leq b_{0} u_{3+} .
$$

By (2.14), (2.21) and (2.24), it follows from a direct computation that

$$
s_{0} \leq \sqrt{\frac{c_{*}^{2}-\mu^{2} q_{0}^{2}}{q_{0}^{2}-c_{*}^{2}}}+\frac{1}{2}\left(1-\mu^{2}\right) q_{0}^{4} b_{0}+O\left(b_{0}^{2}\right) .
$$

From (2.23) and (2.25), then (2.20) holds true.

In addition, substituting (2.20) into (2.14) leads to

$$
U\left(s_{0}\right) \leq q_{0} b_{0}+O\left(b_{0}^{2}\right), \quad u_{3}\left(s_{0}\right)=q_{0}+O\left(b_{0}\right) .
$$

Next, we show that $u_{3}(s)>c(s)$ holds true for $b_{0} \leq s \leq s_{0}$.

By (2.1), we have

$$
\frac{U^{\prime}(s)}{u_{3}^{\prime}(s)}=-\frac{1}{s} \quad \Rightarrow \quad U^{\prime}\left(u_{3}\right)=-\frac{1}{s}
$$


This yields

$$
U\left(u_{3}\left(s_{0}\right)\right)-U\left(u_{3}\left(b_{0}\right)\right)=-\int_{b_{0}}^{s_{0}} \frac{1}{s} d u_{3}(s)=\frac{1}{\tau}\left(u_{3}\left(b_{0}\right)-u_{3}\left(s_{0}\right)\right)
$$

for some $\tau \in\left[b_{0}, s_{0}\right]$.

This, together with $U\left(b_{0}\right)=b_{0} u_{3}\left(b_{0}\right)$ yields

$$
u_{3}\left(b_{0}\right)=\frac{u_{3}\left(s_{0}\right)+\tau U\left(s_{0}\right)}{1+b_{0} \tau} \geq \frac{u_{3}\left(s_{0}\right)}{1+b_{0} s_{0}} .
$$

On the other hand, by Bernoulli's law, one has

$$
c_{*}^{2}=\mu^{2} q_{0}^{2}+\left(1-\mu^{2}\right) c^{2}\left(\rho_{0}\right)=\mu^{2}\left(u_{3}^{2}(s)+U^{2}(s)\right)+\left(1-\mu^{2}\right) c^{2}(s),
$$

i.e.

$$
c^{2}(s)=\frac{1}{1-\mu^{2}} c_{*}^{2}-\frac{\mu^{2}}{1-\mu^{2}}\left(u_{3}^{2}(s)+U^{2}(s)\right) .
$$

Thus, by (2.28) and (2.29), we arrive at

$$
u_{3}^{2}\left(b_{0}\right)-c^{2}\left(b_{0}\right)=\frac{1+b_{0}^{2} \mu^{2}}{1-\mu^{2}} u_{3}^{2}\left(b_{0}\right)-\frac{1}{1-\mu^{2}} c_{*}^{2} \geq \frac{1+b_{0}^{2} \mu^{2}}{1-\mu^{2}} \frac{u_{3}^{2}\left(s_{0}\right)}{\left(1+b_{0} s_{0}\right)^{2}}-\frac{1}{1-\mu^{2}} c_{*}^{2} .
$$

Substituting (2.26) into (2.30) yields

$$
u_{3}^{2}\left(b_{0}\right)-c^{2}\left(b_{0}\right) \geq \frac{1+\mu^{2} b_{0}^{2}}{1-\mu^{2}} \frac{q_{0}^{2}+O\left(b_{0}\right)}{\left(1+b_{0} s_{0}\right)^{2}}-\frac{c_{*}^{2}}{1-\mu^{2}}=\frac{1}{1-\mu^{2}}\left(q_{0}^{2}-c_{*}^{2}\right)+O\left(b_{0}\right) .
$$

Then for small $b_{0}$, we obtain

$$
u_{3}^{2}\left(b_{0}\right)-c^{2}\left(b_{0}\right)>0 .
$$

In addition, it follows from (i) of Lemma 2.2 and a direct calculation that

$$
\left(u_{3}(s)-c\left(\rho(s), S_{+}\right)\right)^{\prime}=u_{3}^{\prime}(s)-c_{\rho}^{\prime}\left(\rho, S_{+}\right) \rho^{\prime}(s)>0 .
$$

This yields

$$
u_{3}(s)>c(s), \quad \text { for } s \in\left[b_{0}, s_{0}\right] .
$$

(iii). Since $\lambda_{3}^{\prime}(s)=\frac{\partial \lambda_{3}}{\partial U} U^{\prime}(s)+\frac{\partial \lambda_{3}}{\partial u_{3}} u_{3}^{\prime}(s)+\frac{\partial \lambda_{3}}{\partial c} c_{\rho}^{\prime}\left(\rho, S_{+}\right) \rho^{\prime}(s)$, and by use of (ii) of Lemma 2.2 , then we arrive at

$$
\left\{\begin{array}{l}
\frac{\partial \lambda_{3}}{\partial U}=\frac{1}{u_{3}^{2}-c^{2}}\left(u_{3}+\frac{c U}{\sqrt{U^{2}+u_{3}^{2}-c^{2}}}\right)>0, \\
\frac{\partial \lambda_{3}}{\partial u_{3}}=-\frac{1}{\left(u_{3}^{2}-c^{2}\right)^{2}}\left(U\left(u_{3}^{2}+c^{2}\right)+\frac{2 c U^{2} u_{3}+c u_{3}\left(u_{3}^{2}-c^{2}\right)}{\sqrt{U^{2}+u_{3}^{2}-c^{2}}}\right)<0, \\
\frac{\partial \lambda_{3}}{\partial c}=\frac{2 c U u_{3}}{\left(u_{3}^{2}-c^{2}\right)^{2}}+\frac{\sqrt{U^{2}+u_{3}^{2}-c^{2}}}{u_{3}^{2}-c^{2}}+\frac{2 c^{2} U^{2}+c^{2}\left(u_{3}^{2}-c^{2}\right)}{\left(u_{3}^{2}-c^{2}\right)^{2} \sqrt{U^{2}+u_{3}^{2}-c^{2}}}>0 .
\end{array}\right.
$$

Then together with $U^{\prime}(s)<0, u_{3}^{\prime}(s)>0$ and $\rho^{\prime}(s)<0$, we obtain $\lambda_{3}^{\prime}(s)<0$ for $b_{0} \leq s \leq$ $s_{0}$. Combining with the entropy condition (2.4) yields $\lambda_{3}(s) \geq \lambda_{3}\left(s_{0}\right)>s_{0}$.

REMARK 2.1. Since the denominator of the system (2.1) is positive in $\left[b_{0}, s_{0}\right]$, then by (2.1) we can smoothly extend the background solution $\left(\tilde{\rho}(s), \tilde{U}(s), \tilde{u}_{3}(s), \tilde{P}(s)\right)$ of $(2.1)$ (2.5) to some interval $\left[b_{0}, s_{0}+\tau_{0}\right]$ with $\tau_{0}>0$. The related extensions are denoted by $\left(\hat{\rho}(s), \hat{U}(s), \hat{u}_{3}(s), \hat{P}(s)\right)$. 
3. Some crucial properties on globally stable supersonic conic waves. In this section, under the assumptions of Theorem 1.1, we focus on some basic observations on the globally stable supersonic conic shock wave for the full Euler system as in [15.

It is noted that it follows from the first equation of (1.4) that one can introduce a stream function $\psi\left(x_{3}, r\right)$ with $\psi(0,0)=0$ such that $\partial_{x_{3}} \psi\left(x_{3}, r\right)=-r \rho^{+} U^{+}$and $\partial_{r} \psi\left(x_{3}, r\right)=r \rho^{+} u_{3}^{+}$.

In addition, by (1.4)-(1.5) and the state equation, we can easily deduce that $\left(U^{+} \partial_{r}+\right.$ $\left.u_{3}^{+} \partial_{x_{3}}\right)\left(\frac{P^{+}}{\left(\rho^{+}\right)^{\gamma}}\right)=0$. This means that $\frac{P^{+}}{\left(\rho^{+}\right)^{\gamma}}=$ constant holds true along each stream line (the global existence of a stream line for $x_{3}>0$ is guaranteed by (ii) of Theorem 1.1 ), which is denoted by a function $Q$. Namely, $Q$ can be expressed as

$$
Q=Q(\psi)
$$

Next, we derive a crucial relation between $Q(\psi)$ and the rotation $\partial_{3} U^{+}-\partial_{r} u_{3}^{+}$.

Substituting the first equation of (1.4) and the expression $P^{+}=Q\left(\psi\left(x_{3}, r\right)\right)\left(\rho^{+}\right)^{\gamma}$ into the second and the third equations in (1.4) yield respectively

$$
\left\{\begin{array}{l}
\partial_{r}\left(r \rho^{+} U^{+}\right)+\partial_{3}\left(r \rho^{+} u_{3}^{+}\right)=0 \\
\rho^{+} U^{+} \partial_{r} U^{+}+\rho^{+} u_{3}^{+} \partial_{3} U^{+}+\partial_{r} P^{+}=0 \\
\rho^{+} U^{+} \partial_{r} u_{3}^{+}+\rho^{+} u_{3}^{+} \partial_{3} u_{3}^{+}+\partial_{3} P^{+}=0
\end{array}\right.
$$

and

$$
\left\{\begin{array}{l}
\partial_{r}\left(r \rho^{+} U^{+}\right)+\partial_{3}\left(r \rho^{+} u_{3}^{+}\right)=0 \\
U^{+} \partial_{r} U^{+}+u_{3}^{+} \partial_{3} U^{+}+Q^{\prime}(\psi)\left(\rho^{+}\right)^{\gamma-1} \partial_{r} \psi+\gamma Q(\psi)\left(\rho^{+}\right)^{\gamma-2} \partial_{r} \rho^{+}=0 \\
U^{+} \partial_{r} u_{3}^{+}+u_{3}^{+} \partial_{3} u_{3}^{+}+Q^{\prime}(\psi)\left(\rho^{+}\right)^{\gamma-1} \partial_{3} \psi+\gamma Q(\psi)\left(\rho^{+}\right)^{\gamma-2} \partial_{3} \rho^{+}=0 .
\end{array}\right.
$$

In addition, it follows from Bernoulli's law that

$$
U^{+} \partial_{r} U^{+}+u_{3}^{+} \partial_{r} u_{3}^{+}+\gamma Q(\psi)\left(\rho^{+}\right)^{\gamma-2} \partial_{r} \rho^{+}+\gamma\left(\rho^{+}\right)^{\gamma-1} Q^{\prime}(\psi) \partial_{r} \psi=0 .
$$

This, together with the second equation in (3.3), yields

$$
Q^{\prime}(\psi)=\frac{\partial_{3} U^{+}-\partial_{r} u_{3}^{+}}{(\gamma-1) r\left(\rho^{+}\right)^{\gamma}}
$$

Thus, one has

$$
\lim _{x_{3} \rightarrow \infty}\left(\partial_{3} U^{+}-\partial_{r} u_{3}^{+}\right)\left(x_{3}, r\left(x_{3}, x_{3}^{0}\right)\right)=\infty \quad \text { if } Q^{\prime}\left(\psi\left(x_{3}^{0}, \chi\left(x_{3}^{0}\right)\right)\right) \neq 0 \text { with } x_{3}^{0}>0 ;
$$

here $r\left(x_{3}, x_{3}^{0}\right)$ stands for the stream line starting from the point $\left(x_{3}^{0}, \chi\left(x_{3}^{0}\right)\right)$ at the shock curve, i.e., which is determined by

$$
\frac{d r\left(x_{3}, x_{3}^{0}\right)}{d x_{3}}=\left(\frac{U^{+}}{u_{3}^{+}}\right)\left(x_{3}, r\left(x_{3}, x_{3}^{0}\right)\right),\left.\quad r\left(x_{3}, x_{3}^{0}\right)\right|_{x_{3}=x_{3}^{0}}=\chi\left(x_{3}^{0}\right) .
$$

To fulfill (i) and (ii) of Theorem 1.1 , by (3.4)-(3.5), it is required that $\left(\partial_{3} U^{+}-\right.$ $\left.\partial_{r} u_{3}^{+}\right)\left(x_{3}, \chi\left(x_{3}\right)\right) \equiv 0$ must hold true for $x_{3}>0$. Furthermore, we have

LEMma 3.1. Under the assumptions of Theorem 1.1, for $x_{3}>0,\left(\partial_{3} U^{+}-\partial_{r} u_{3}^{+}\right)\left(x_{3}, \chi\left(x_{3}\right)\right)$ $\equiv 0$ holds true if and only if $\chi^{\prime \prime}\left(x_{3}\right) \equiv 0$. 
REMARK 3.1. By Lemma 3.1, it is easy to show that $\left(\partial_{3} U^{+}-\partial_{r} u_{3}^{+}\right)\left(x_{3}, \chi\left(x_{3}\right)\right) \equiv 0$ for $x_{3}>0$ if and only if $\chi\left(x_{3}\right)=s_{0} x_{3}$ due to $\chi(0)=0$ and $\chi^{\prime}(0)=s_{0}$.

Proof. It follows from (1.6) that on $r=\chi\left(x_{3}\right)$,

$$
\left\{\begin{array}{l}
\rho^{+} U^{+}-\rho^{+} u_{3}^{+} \chi^{\prime}=-\rho_{0} q_{0} \chi^{\prime}, \\
P^{+}+\rho^{+}\left(U^{+}\right)^{2}-\chi^{\prime} \rho^{+} U^{+} u_{3}^{+}=P_{0}, \\
\rho^{+} U^{+} u_{3}^{+}-P^{+} \chi^{\prime}-\rho^{+}\left(u_{3}^{+}\right)^{2} \chi^{\prime}=-P_{0} \chi^{\prime}\left(x_{3}\right)-\rho_{0} q_{0}^{2} \chi^{\prime} .
\end{array}\right.
$$

Multiplying by $\chi^{\prime}$ on both sides of the second equation in (3.6) and then adding the third equation in (3.6) yields

$$
\left(U^{+} \chi^{\prime}+u_{3}^{+}\right)\left(\rho^{+} U^{+}-\rho^{+} u_{3}^{+} \chi^{\prime}\right)=-\rho_{0} q_{0}^{2} \chi^{\prime} .
$$

From this, together with the first equation in (3.6) and the fact that $\chi^{\prime} \neq 0$, we obtain on $r=\chi\left(x_{3}\right)$,

$$
U^{+} \chi^{\prime}+u_{3}^{+}=q_{0}
$$

Taking $\partial_{x_{3}}$ on (3.7) yields

$$
\chi^{\prime} \partial_{3} U^{+}+U^{+} \chi^{\prime \prime}+\partial_{3} u_{3}^{+}+\left(\chi^{\prime}\right)^{2} \partial_{r} U^{+}+\partial_{r} u_{3}^{+} \chi^{\prime}=0 \quad \text { on } r=\chi\left(x_{3}\right) .
$$

In addition, by differentiating the second equation of (3.6) with respect to the variable $x_{3}$, we arrive at

$$
\partial_{3} P^{+}+\chi^{\prime} \partial_{r} P^{+}+\partial_{3}\left(U^{+}\left(\rho^{+} U^{+}-\rho^{+} u_{3}^{+} \chi^{\prime}\right)\right)+\chi^{\prime} \partial_{r}\left(U^{+}\left(\rho^{+} U^{+}-\rho^{+} u_{3}^{+} \chi^{\prime}\right)\right)=0 .
$$

It follows from the second equation, the third equation of (3.6) and (3.9) that

$$
\begin{aligned}
& -\rho^{+} U^{+} \partial_{r} u_{3}^{+}-\rho^{+} u_{3}^{+} \partial_{3} u_{3}^{+}-\rho^{+} u_{3}^{+}\left(\chi^{\prime}\right)^{2} \partial_{r} U^{+}+\partial_{3} U^{+}\left(\rho^{+} U^{+}-2 \rho^{+} u_{3}^{+} \chi^{\prime}\right) \\
& +U^{+} \frac{d}{d x_{3}}\left(\rho^{+} U^{+}-\rho^{+} u_{3}^{+} \chi^{\prime}\right)=0 .
\end{aligned}
$$

This, together with the first equation in (3.6), yields

$$
-\rho^{+} U^{+} \partial_{r} u_{3}^{+}-\rho^{+} u_{3}^{+} \partial_{3} u_{3}^{+}-\rho^{+} u_{3}^{+}\left(\chi^{\prime}\right)^{2} \partial_{r} U^{+}+\partial_{3} U^{+}\left(\rho^{+} U^{+}-2 \rho^{+} u_{3}^{+} \chi^{\prime}\right)=\rho_{0} q_{0} U^{+} \chi^{\prime \prime} \text {. }
$$

Combining (3.8) with (3.10) yields

$$
\left(\rho^{+} U^{+}-\rho^{+} u_{3}^{+} \chi^{\prime}\right)\left(\partial_{3} U^{+}-\partial_{r} u_{3}^{+}\right)=\left(\rho_{0} q_{0}-\rho^{+} u_{3}^{+}\right) U^{+} \chi^{\prime \prime} .
$$

Thanks to the first equation of (3.6), one has on $r=\chi\left(x_{3}\right)$,

$$
\left(\partial_{3} U^{+}-\partial_{r} u_{3}^{+}\right) \rho_{0} q_{0} \chi^{\prime}=\rho^{+}\left(U^{+}\right)^{2} \frac{\chi^{\prime \prime}}{\chi^{\prime}} .
$$

Due to $\chi^{\prime}\left(x_{3}\right) \neq 0$ and $U^{+} \neq 0$, then we have

$$
\partial_{3} U^{+}-\partial_{r} u_{3}^{+}=0 \Leftrightarrow \chi^{\prime \prime}=0 \quad \text { on } r=\chi\left(x_{3}\right) .
$$

Thus, we complete the proof of Lemma 3.1.

In the next section, based on Lemma 3.1 and Remark 3.1, we will complete the proof of Theorem 1.1. 
4. Proof of Theorem 1.1. By Lemma 3.1 and Remark 3.1, we know that the shock curve must be straight, whose slope is just only $s_{0}$ in terms of Remark 1.1. Together with the expression $Q^{\prime}(\psi)=\frac{\partial_{3} U^{+}-\partial_{r} u_{3}^{+}}{(\gamma-1) r\left(\rho^{+}\right)^{\gamma}}$, then one has $Q^{\prime}(\psi) \equiv 0$. This implies that $Q(\psi) \equiv$ constant holds true behind the shock $r=s_{0} x_{3}$, which is denoted by a positive constant $A$. Therefore, we have

$$
P^{+}\left(x_{3}, r\right)=A\left(\rho^{+}\right)^{\gamma}\left(x_{3}, r\right) \quad \text { and } \quad \partial_{3} U^{+}-\partial_{r} u_{3}^{+} \equiv 0 .
$$

From this, it is easy to show that the supersonic flow field behind the shock can be described by the potential flow equation. We now set $\partial_{r} \phi=U^{+}$and $\partial_{x_{3}} \phi=u_{3}^{+}$with $\phi(0,0)=0$. Then it follows from the second and third equations in (3.2) and the state equation of polytropic flow that

$$
\frac{1}{2}|\nabla \phi|^{2}+h\left(\rho^{+}\right)=C_{0}
$$

here $h\left(\rho^{+}\right)=\frac{c^{2}\left(\rho^{+}\right)}{\gamma-1}$ is the specific enthalpy.

By use of (4.2) and the implicit function theorem, it is easy to see that the density function $\rho^{+}(x)$ can be expressed as

$$
\rho^{+}=h^{-1}\left(C_{0}-\frac{1}{2}|\nabla \phi|^{2}\right) \equiv H(\nabla \phi) .
$$

Substituting (4.3) into the first equation in (3.2) yields

$$
\left(c^{2}(\nabla \phi)-\left(\partial_{3} \phi\right)^{2}\right) \partial_{3}^{2} \phi-2 \partial_{3} \phi \partial_{r} \phi \partial_{r x_{3}}^{2} \phi+\left(c^{2}(\nabla \phi)-\left(\partial_{r} \phi\right)^{2}\right) \partial_{r}^{2} \phi+\frac{c^{2}(\nabla \phi)}{r} \partial_{r} \phi=0
$$

here $c(\nabla \phi) \equiv c(H(\nabla \phi))$.

Next, we look for the value of $\phi$ on the shock.

On $r=s_{0} x_{3}$, we have

$$
\begin{aligned}
& \phi\left(x_{3}, s_{0} x_{3}\right)=\int_{0}^{x_{3}} \frac{d}{d x_{3}}\left(\phi\left(x_{3}, s_{0} x_{3}\right)\right) d x_{3} \\
& =\int_{0}^{x_{3}}\left(u_{3}\left(s_{0}\right)+U\left(s_{0}\right) s_{0}\right) d x_{3} \\
& =\left(u_{3}\left(s_{0}\right)+U\left(s_{0}\right) s_{0}\right) x_{3} \equiv \varphi_{0}\left(x_{3}\right)
\end{aligned}
$$

and

$\partial_{\vec{n}} \phi\left(x_{3}, s_{0} x_{3}\right) \equiv\left(u_{3}\left(s_{0}\right), U\left(s_{0}\right)\right) \cdot\left(\frac{s_{0}}{\sqrt{1+s_{0}^{2}}},-\frac{1}{\sqrt{1+s_{0}^{2}}}\right)=\frac{s_{0} u_{3}\left(s_{0}\right)-U\left(s_{0}\right)}{\sqrt{1+s_{0}^{2}}} \equiv \varphi_{1}\left(x_{3}\right)$.

In addition, on the conic surface $r=b\left(x_{3}\right), \phi$ satisfies

$$
\partial_{r} \phi=b^{\prime}\left(x_{3}\right) \partial_{3} \phi .
$$

Let $\Omega_{1} \equiv\left\{\left(x_{3}, r\right): b\left(x_{3}\right)<r<s_{0} x_{3}, x_{3}>0\right\}$ and $\Omega_{2} \equiv\left\{\left(x_{3}, r\right): b_{0} x_{3}<r<\right.$ $\left.s_{0} x_{3}, x_{3}>0\right\}$ be domains. Under the assumptions of Theorem 1.1, we know that the related potential function $\phi\left(x_{3}, r\right) \in \operatorname{Lip}\left(\bar{\Omega}_{1}\right) \cap C^{2}\left(\bar{\Omega}_{1} \backslash(0,0)\right)$. From this, we can derive that 
Lemma 4.1. Under the assumptions of Theorem 1.1, the solution $\left(\rho^{+}, U^{+}, u_{3}^{+}, P^{+}\right)$is the same as the background solution $\left(\tilde{\rho}\left(\frac{r}{x_{3}}\right), \tilde{U}\left(\frac{r}{x_{3}}\right), \tilde{u}_{3}\left(\frac{r}{x_{3}}\right), \tilde{P}\left(\frac{r}{x_{3}}\right)\right)$ in the domain $\bar{\Omega}_{1} \cap \bar{\Omega}_{2}$.

Proof. We now take a rotational transformation as follows:

$$
\left\{\begin{array}{l}
y_{1}=\cos \theta_{0} x_{3}-\sin \theta_{0} r \\
y_{2}=\sin \theta_{0} x_{3}+\cos \theta_{0} r
\end{array}\right.
$$

where $\theta_{0}=\frac{\pi}{2}-\arctan s_{0}$.

In this case, the shock curve $r=s_{0} x_{3}$ is changed into $y_{1}=0$, and the nonlinear problem (4.4)-(4.7) can be rewritten as

$$
\left\{\begin{array}{c}
\left(1-\frac{\left(\partial_{y_{1}} \varphi\right)^{2}}{c^{2}\left(\nabla_{y} \varphi\right)}\right) \partial_{y_{1}}^{2} \varphi-\frac{2 \partial_{y_{1}} \varphi \partial_{y_{2}} \varphi}{c^{2}\left(\nabla_{y} \varphi\right)} \partial_{y_{1} y_{2}}^{2} \varphi+\left(1-\frac{\left(\partial_{y_{2}} \varphi\right)^{2}}{c^{2}\left(\nabla_{y} \varphi\right)}\right) \partial_{y_{2}}^{2} \varphi \\
+\frac{\cos \theta_{0} \partial_{y_{2}} \varphi-\sin \theta_{0} \partial_{y_{1}} \varphi}{\cos \theta_{0} y_{2}-\sin \theta_{0} y_{1}}=0 \quad \text { in } \tilde{\Omega}_{1} \\
\varphi\left(0, y_{2}\right)=\left(u_{3}\left(s_{0}\right)+U\left(s_{0}\right) s_{0}\right) \sin \theta_{0} y_{2} \equiv \tilde{\varphi}_{1}\left(y_{2}\right), \\
\partial_{y_{1}} \varphi\left(0, y_{2}\right)=s_{0} u_{3}\left(s_{0}\right)-U\left(s_{0}\right) \equiv \tilde{\varphi}_{2}\left(y_{2}\right), \\
\cos \theta_{0} \partial_{y_{2}} \varphi-\sin \theta_{0} \partial_{y_{1}} \varphi=\left(\cos \theta_{0} \partial_{y_{1}} b+\sin \theta_{0} \partial_{y_{2}} b\right)\left(\cos \theta_{0} \partial_{y_{1}} \varphi+\sin \theta_{0} \partial_{y_{2}} \varphi\right) \\
\text { on } y_{2}=\tan \theta_{0} y_{1}+\sec \theta_{0} b\left(\cos \theta_{0} y_{1}+\sin \theta_{0} y_{2}\right)
\end{array}\right.
$$

here $\varphi(y) \equiv \phi\left(\cos \theta_{0} y_{1}+\sin \theta_{0} y_{2},-\sin \theta_{0} y_{1}+\cos \theta_{0} y_{2}\right)$ and $\tilde{\Omega}_{1} \equiv\left\{\left(y_{1}, y_{2}\right): 0<y_{1}<\right.$ $\left.\cot \theta_{0} y_{2}-\csc \theta_{0} b\left(\cos \theta_{0} y_{1}+\sin \theta_{0} y_{2}\right)\right\}$.

Let $\tilde{\phi}\left(x_{3}, r\right)$ be the potential function of $\left(\tilde{U}\left(\frac{r}{x_{3}}\right), \tilde{u}_{3}\left(\frac{r}{x_{3}}\right)\right)$ with $\tilde{\phi}(0,0)=0$. Under the transformation (4.8), we suppose that $\tilde{\phi}\left(x_{3}, r\right)$ is changed into the function $\tilde{\varphi}(y)$. Then $\tilde{\varphi}(y)$ satisfies

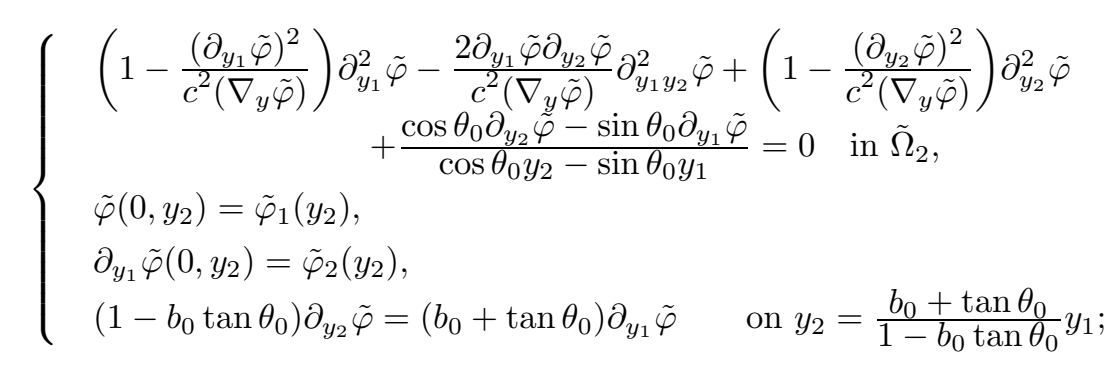

here $\tilde{\varphi}(y) \equiv \tilde{\phi}\left(\cos \theta_{0} y_{1}+\sin \theta_{0} y_{2},-\sin \theta_{0} y_{1}+\cos \theta_{0} y_{2}\right)$ and $\tilde{\Omega}_{2} \equiv\left\{\left(y_{1}, y_{2}\right): 0<y_{1}<\right.$ $\left.\frac{1-b_{0} \tan \theta_{0}}{b_{0}+\tan \theta_{0}} y_{2}\right\}$.

Next, we prove that $\varphi=\tilde{\varphi}$ in $\overline{\tilde{\Omega}_{1} \cap \tilde{\Omega}_{2}}$.

Set $w=\varphi-\tilde{\varphi}$. Then it follows from (4.9)-(4.10) that $w$ satisfies

$$
\left\{\begin{array}{l}
\sum_{i, j=1}^{2} a_{i j}(y) \partial_{y_{i} y_{j}}^{2} w+\sum_{i=1}^{2} b_{i}(y) \partial_{y_{i}} w=0 \quad \text { in } \tilde{\Omega}_{1} \cap \tilde{\Omega}_{2} \\
w\left(0, y_{2}\right)=0 \\
\partial_{y_{1}} w\left(0, y_{2}\right)=0
\end{array}\right.
$$


here

$$
a_{11}(y)=1-\frac{\left(\partial_{y_{1}} \varphi\right)^{2}}{c^{2}\left(\nabla_{y} \varphi\right)}, \quad a_{12}(y)=a_{21}(y)=-\frac{\partial_{y_{1}} \varphi \partial_{y_{2}} \varphi}{c^{2}\left(\nabla_{y} \varphi\right)}, \quad a_{22}(y)=1-\frac{\left(\partial_{y_{2}} \varphi\right)^{2}}{c^{2}\left(\nabla_{y} \varphi\right)},
$$

and $b_{i}(y)(i=1,2)$ are continuous functions in $\tilde{\Omega}_{1} \cap \tilde{\Omega}_{2}$.

Noting that

$$
\begin{aligned}
\left(\frac{2 \partial_{y_{1}} \tilde{\varphi} \partial_{y_{2}} \tilde{\varphi}}{c^{2}\left(\nabla_{y} \tilde{\varphi}\right)}\right)^{2}-4\left(1-\frac{\left(\partial_{y_{1}} \tilde{\varphi}\right)^{2}}{c^{2}\left(\nabla_{y} \tilde{\varphi}\right)}\right)\left(1-\frac{\left(\partial_{y_{2}} \tilde{\varphi}\right)^{2}}{c^{2}\left(\nabla_{y} \tilde{\varphi}\right)}\right) \\
=\frac{4}{c^{2}\left(\nabla_{y} \tilde{\varphi}\right)}\left(\left(\partial_{y_{1}} \tilde{\varphi}\right)^{2}+\left(\partial_{y_{2}} \tilde{\varphi}\right)^{2}-c^{2}\left(\nabla_{y} \tilde{\varphi}\right)\right)>0
\end{aligned}
$$

and

$c^{2}\left(\nabla_{y} \tilde{\varphi}\right)-\left(\partial_{y_{1}} \tilde{\varphi}\right)^{2}=-\frac{1}{1+s_{0}^{2}}\left(\left(\tilde{u}_{3}^{2}-c^{2}\right) s_{0}^{2}-2 \tilde{u}_{3} \tilde{U} s_{0}+\left(\tilde{U}^{2}-c^{2}\right)\right)>0$ due to Lemma 2.2(iii), thus, together with (ii) of Theorem 1.1, we have

$$
a_{11}>0, \quad a_{12}<0 \quad \text { and } \quad \Delta=4\left(a_{12}^{2}-a_{11} a_{22}\right)>0 \quad \text { in } \quad \overline{\Omega_{1} \cap \tilde{\Omega}_{2}} .
$$

This means that (4.11) is strictly hyperbolic with respect to the $y_{1}$ direction. By the standard result on the existence and uniqueness of solutions to hyperbolic equations (one can see the proof procedure of Theorem 4.1 in [1] due to $a_{i j}(y) \in C^{1}\left(\overline{\tilde{\Omega}_{1} \cap \tilde{\Omega}_{2}} \backslash(0,0)\right)$, $b_{i}(y) \in C\left(\overline{\tilde{\Omega}_{1} \cap \tilde{\Omega}_{2}} \backslash(0,0)\right)$ and Remark 1.1), we know that $w \equiv 0$ in $\overline{\tilde{\Omega}_{1} \cap \tilde{\Omega}_{2}}$. Thus, returning to the original coordinates $\left(x_{3}, r\right)$, we can complete the proof of Lemma 4.1.

Based on Lemma 4.1, we now show the following conclusion. From this, Theorem 1.1 will be easily derived.

Theorem 4.2. Under the assumptions of Theorem 1.1, $\Omega_{1}=\Omega_{2}$ holds true. Moreover, $\phi\left(x_{3}, r\right) \equiv \tilde{\phi}\left(x_{3}, r\right)$.

Proof. Set

$$
\begin{aligned}
& \Gamma_{b} \equiv\left\{\left(x_{3}, r\right): r=b\left(x_{3}\right), x_{3}>0\right\}, \\
& L \equiv\left\{\left(x_{3}, r\right): r=b_{0} x_{3}, x_{3}>0\right\} .
\end{aligned}
$$

Due to $b\left(x_{3}\right)=b_{0} x_{3}+\varepsilon \varphi\left(x_{3}\right)$ with $\varphi\left(x_{3}\right) \in C_{0}^{\infty}(0, l)$, then we can conclude that there exist at least two intersection points $P_{1}$ and $P_{2}$ in $\Gamma_{b} \cap L$ such that the $\operatorname{arc} P_{1} P_{2}$ lies above $L$ or lies under $L$. Without loss of generality, we assume that the $\operatorname{arc} P_{1} P_{2}$ lies above $L$. In addition, for convenience, the coordinate of $P_{1}$ is denoted by $\left(x_{3}^{P_{1}}, r^{P_{1}}\right)$ (see Figure (5).

Consider the following stream line starting from $P_{1}$ :

$$
\left\{\begin{array}{l}
\frac{d r}{d x_{3}}=\frac{\partial_{r} \tilde{\phi}\left(x_{3}, r\right)}{\partial_{3} \tilde{\phi}\left(x_{3}, r\right)} \\
r\left(x_{3}^{P_{1}}\right)=r^{P_{1}}
\end{array}\right.
$$

Due to Lemma 4.1, we know that there exist two different stream lines $r=b_{0} x_{3}$ and $r=b\left(x_{3}\right)$ between $P_{1}$ and $P_{2}$ for the system (1.4). However, this is contradictory with the uniqueness of the $C^{1}$ solution to the ordinary differential equation (4.13). Thus, we must have $\Omega_{1}=\Omega_{2}$. Consequently, the proof of Theorem 4.1 is completed. 


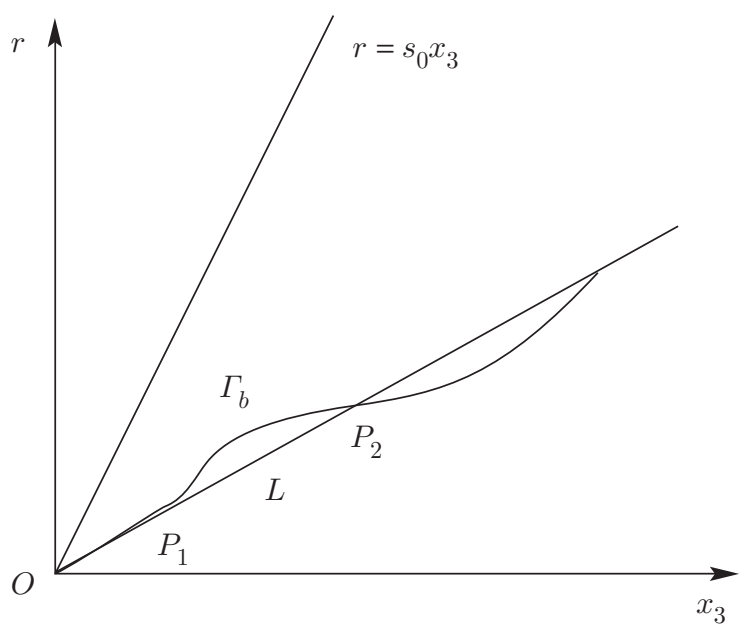

FIG. 5

Based on Theorem 4.1, we now start to prove Theorem 1.1.

Proof of Theorem 1.1. By use of Theorem 4.1, $\Omega_{1} \equiv \Omega_{2}$ holds true. However, the boundary $r=b\left(x_{3}\right)$ is obviously different from the boundary $r=b_{0} x_{3}$. Thus, under the assumptions of Theorem 1.1, a globally stable supersonic shock solution does not exist.

Acknowledgments. The authors would like to thank the referee very much for his (or her) many suggestions which led to substantial improvements of the presentation.

\section{REFERENCES}

[1] S. Alinhac, Temps de vie des solutions régulières des équations d'Euler compressibles axisymétriques en dimension deux, Invent. Math. 111, no. 3, 627-670, 1993. MR.1202138 (94a:35089)

[2] Chen Shuxing, Li Dening, Conical shock waves for an isentropic Euler system, Proc. Roy. Soc. Edinburgh Sect. A 135, no. 6, 1109-1127, 2005. MR2191891 (2006i:35243)

[3] Chen Shuxing, Xin Zhouping, Yin Huicheng, Global shock wave for the supersonic flow past a perturbed cone, Comm. Math. Phys., 228, 47-84, 2002. MR.1911248(2003c:76076)

[4] R.Courant, K.O.Friedrichs, Supersonic flow and shock waves, Interscience Publishers Inc., New York, 1948. MR0029615 (10:637c)

[5] Cui Dacheng Cui, Yin Huicheng, Global supersonic conic shock wave for the steady supersonic flow past a cone: Polytropic case, J. Differential Equations, 246, 641-669, 2009. MR2468732 (2009h:35261)

[6] Cui Dacheng, Yin Huicheng, Global conic shock wave for the steady supersonic flow past a cone: Isothermal case, Pacific J. Math., 233, no. 2, 257-289, 2008. MR2366376 (2009b:76143)

[7] P. Godin, The lifespan of a class of smooth spherically symmetric solutions of the compressible Euler equations with variable entropy in three space dimensions, Arch. Ration. Mech. Anal. 177, no. 3, 479-511, 2005. MR2187620(2006i:76092)

[8] P. Godin, Global shock waves in some domains for the isentropic irrotational potential flow equations, Comm. Partial Differential Equations, Vol.22, no. 11-12, 1929-1997, 1997. MR1629510 (99i:35097)

[9] F. John, Nonlinear wave equations, formation of singularities, University Lecture Series 2, American Mathematical Society, Providence, RI, 1990. MR1066694 (91g:35001) 
[10] T. C. Sideris, Formation of singularities in three-dimensional compressible fluids, Comm. Math. Phys. 101, no. 4, 475-485, 1985. MR815196 (87d:35127)

[11] J. A. Smoller, Shock waves and reaction-diffusion equations, Berlin-Heidelberg-New York, SpringerVerlag, New York, 1983. MR0688146 (84d:35002)

[12] A. Majda, The existence of multidimensional shock fronts, Mem. Amer. Math. Soc. 43, no. 281, 1983. MR699241 (85f:35139)

[13] Xin Zhouping, Yin Huicheng, Global multidimensional shock wave for the steady supersonic flow past a three-dimensional curved cone, Analysis and Applications, Vol.4, No. 2, 101-132, 2006. MR2216450(2007e:35193)

[14] Xu Gang, Yin Huicheng, Instability of one global transonic shock wave for the steady supersonic Euler flow past a sharp cone, Nagoya J. Math., Vol. 199, 151-181, 2010. MR.2732336

[15] Yin Huicheng, Formation and construction of a shock wave for 3-D compressible Euler equations with the spherical initial data, Nagoya Math. J. 175, 125-164, 2004. MR2085314 (2005f:35203) 